\title{
Editorial
}

\author{
Jorge C. S. Cardoso \\ CISUC/Department of Informatics Engineering \\ University of Coimbra \\ Portugal \\ CITAR/School of Arts \\ Portuguese Catholic University \\ Portugal \\ jorgecardoso@ieee.org
}

In last year's editorial, I described the various evolution phases through which the Journal of Science and Technology of the Arts (CITARJ) had gone since its inception in 2008. This year of 2018 marks the $10^{\text {th }}$ volume of CITARJ and also the beginning of a new phase: the editorial team (editor and associate editors) is stepping down and a new team is taking over CITARJ. I am sure that the energy of the new team will keep CITARJ on the right track and push it to new levels of reputation, and scientific and editorial rigor.

CITARJ is now an established scientific journal. It has published an average of 16 articles+reviews per year in the last 5 years (compared to an average of 9 in the previous 5 years) - see Chart 1. It is indexed in DOAJ, Scopus, and Web of Science Core Collection - Emerging Sources Citation Index. It is now also listed in Scimago Journal \& Country Rank and ranked in Quartile 2 for the "Visual Arts and Performing Arts" category.

CITARJ's impact has also been steadily increasing. The average number of citations to our articles is now at about 0.3 citations per article (internal citations are residual as can be verified in Chart 2). Impact can also be observed in the growing number of full text downloads of our articles. Since we have started measuring in 2015, downloads have increased from about 1500 in 2015 to more than 4000 in 2018 (Chart 3).

Although we can consider CITARJ as an established journal, this does not mean that the new editorial team that is taking over in January 2019 can rest. Scientific publication is, perhaps now more than ever, a tricky business. Competition by commercial publishers is fierce and is now polluted with "predatory" publishers that could not care less about science, ethics, or rigor. Still, they are out there and constantly preying through our inboxes. CITARJ must find ways to reach new readers, authors, and editors while still abiding by privacy preserving regulations. CITARJ must continue its growth course in order to stay relevant both internally (within CITAR and the Portuguese Catholic University) and externally (nationally and internationally). This may require a different resource management strategy one that encourages and recognizes volunteer work by the faculty of the School of Arts.

In this issue of CITARJ, Rita Xavier Monteiro and Helena Barranha discuss affectivity in the context of Post-Internet art. António Bandeira Araújo teaches you how to manually draw equirectangular virtual reality panoramas using a ruler, compass, and protractor. Mei-Kei Lai reviews how scent has been treated as an aesthetic medium in art and discusses opportunities for the use of olfactory displays. Pedro Pinto Neves, Leonel Morgado, and Nelson Zagalo propose a new model for agency in videogames using the concept of bio-costs contract. Finally, Kendra Chilson, and Máté Szabó provide us with a review of the exhibition "Thinking Machines: Art and Design in the Computer Age, 1959-1989". 


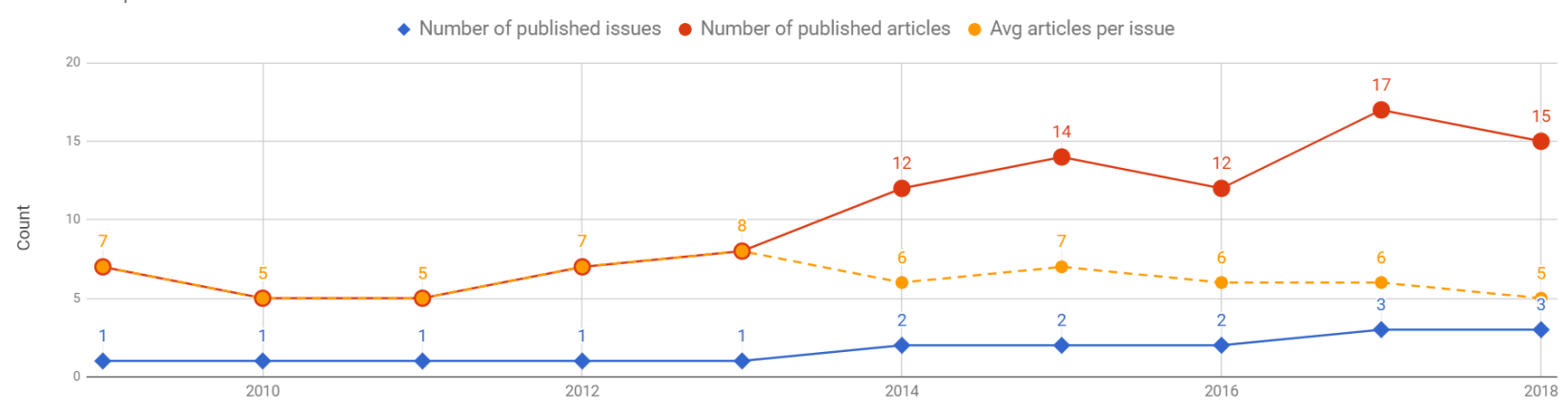

Year

Chart 1 | Number of published issues and articles.

Average citations per article (based on CrossRef data)

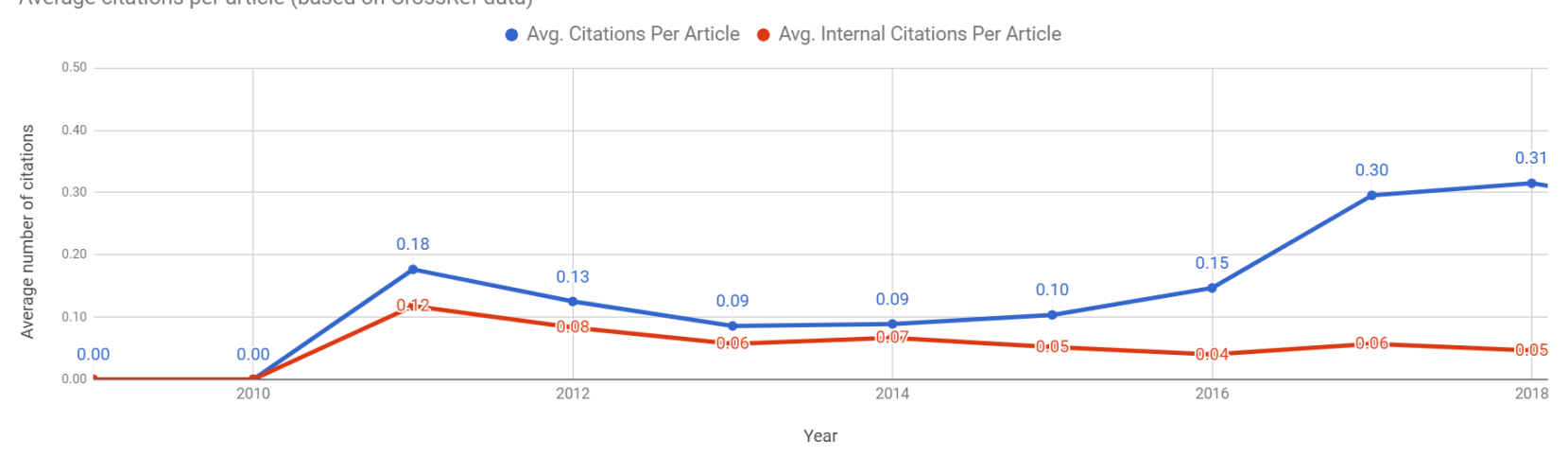

Chart 2 | Average citations per article.

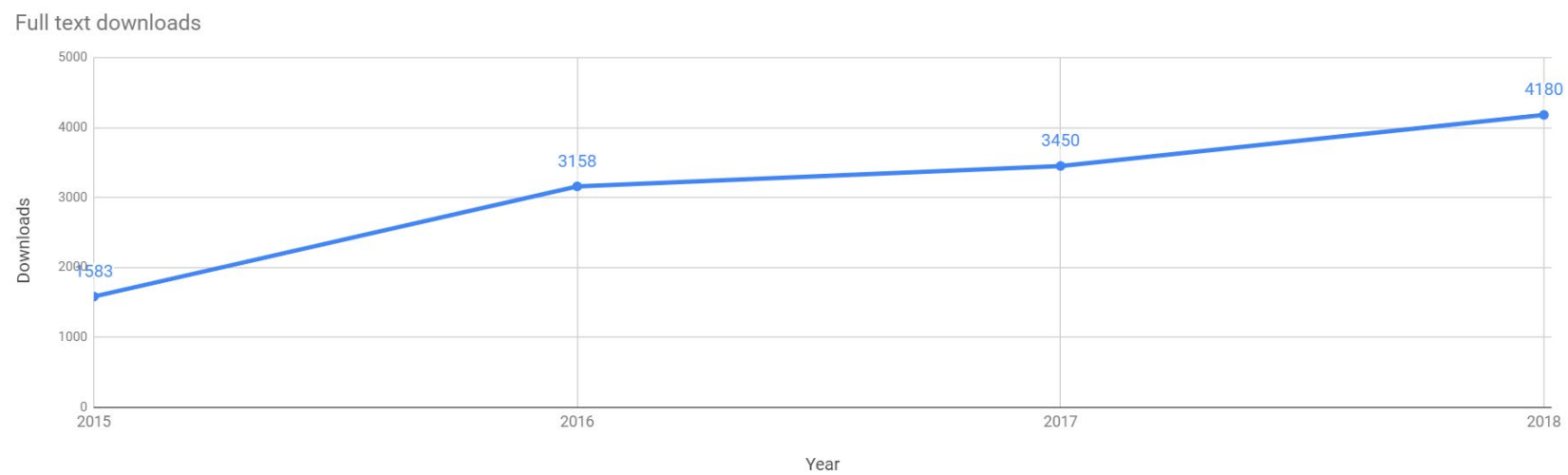

Chart 3 | Full text downloads per year.

\section{BIOGRAPHICAL INFORMATION}

Jorge C. S. Cardoso is Assistant Professor at the University of Coimbra (Department of Informatics Engineering), researcher at the Centre for Informatics and Systems of the University of Coimbra (CISUC) and at the Research Centre in
Science and Technology of the Arts of the Portuguese Catholic University (CITAR). Editor of the Journal of Science and Technology of the Arts (CITARJ). http://jorgecardoso.eu 